to the catheterisation laboratory between 8 and $12 \mathrm{~h}$ after the last subcutaneous dose, designed to receive $60 \mathrm{IU} / \mathrm{kg}$ IV dalteparin $5 \mathrm{~min}$ before PCI, and no additional dalteparin was given to patients who came to the catheterisation laboratory within $8 \mathrm{~h}$ of the last subcutaneous dose. The primary efficacy outcome was the incidence of death, non-fatal myocardial infarction (MI), and urgent revascularization for repeating ischaemia. The primary safety outcome was the incidence of bleeding not related to coronary artery bypass graft (CABG) surgery.

Results One patient developed acute thrombosis during PCI but not resulted in acute myocardial infarction (AMI). In-hospital and during follow-up within 35 days, no patient experienced death or non-fatal myocardial infarction and urgent target vessel revascularization, Thrombolysis In Myocardial Infarction (TIMI) major bleeding and minor bleeding occurred in $0 \%$ and $4.2 \%$ of patients respectively.

Conclusions It may be safe and efficient using dalteparin instead of UFH in selective percutaneous coronary intervention of patients with coronary heart disease.

\section{e0545 RESEARCH ON INHIBITING VASCULAR REMODELLING OF CAPTOPRIL AND XUEFUZHUYU CAPSULE ON THE BASIS OF RESTRAINING ACTIVATION AND MIGRATION OF ADVENTITIAL FIBRIBLASTS}

doi:10.1136/hrt.2010.208967.545

Zhao Mingjing, Wang Jie, Sun Qingqin, Wang Shuoren, Lou Lixia, Lv Xiying, Zhang Dongmei. Key Laboratory of Chinese Internal Medicine, Dongzhimen Hospital affiliated Beijing University of Chinese Medicine, Beijing, China

Background Vascular remodelling is the common pathophysiological basis of artherosclerosis, hypertension and coronary artery disease, et al. It is also one of pathogenesis mechanisms which causes target organs damage and dysfunction. Now, new evidences show that the vascular adventitia may be involved in the formation of vascular remodelling. The activation and migrating to the intima of vascular adventitial fibroblasts is one of the important reasons leading to intimal thickening and stenosis.

Objective To observe inhibition of ACEI drugs Captopril and Chinese medicine Xuefuzhuyu Capsule to vascular remodelling and adventitial fibriblasts activating and migrating.

Methods 1. The intima of left carotid common arteries was denudated by balloon injury. Animals with the percutaneous transluminal angioplasty (PTA) were divided into five groups at random: sham, model, Captopril and Xuefuzhuyu group, which including 8 rats. The injured sections were taken out and made into specimens for HE staining to observe vascular wall thickness, area and vascular stricture rate after rats had been drenched for 28d. 2. It was adopted that primary culture of, vascular adventitial fibroblasts and TGF $\beta 1$ induced $(10 \mathrm{ng} / \mathrm{ml})$ model. Rats were randomly divided into normal group, Captopril group and Xuefuzhuyu group. Arterial blood were collected after drenching drug $7 \mathrm{~d}$ and drug serums effected on the TGF $\beta 1$-induced fibroblasts for $48 \mathrm{~h}$. The $\alpha$-SM-actin expression changes were detected by the western blot method and cell migration rate by cell scratch method.

Results 1. Effects of drugs on indexes of vascular remodelling: Neointimal thickness of Xuefuzhuyu group significantly was reduced than that of model group $(18.92 \pm 4.77 \mu \mathrm{m}$ vs $52.11 \pm 21.65 \mu \mathrm{m}$, $\mathrm{p}<0.01)$, and so did neointimal area $\left(0.048 \pm 0.013 \mathrm{~mm}^{2}\right.$ vs $\left.0.130 \pm 0.049 \mathrm{~mm}^{2}, \mathrm{p}<0.01\right)$. At the same time, vascular stricture rate of Xuefuzhuyu group was obviously decreased compared with model group $(8.910 \pm 2.120$ vs $22.85 \pm 9.763 \%, p<0.01)$. These three indexes of Captopril group were no statistically significant differences through lessening trend. 2. Effect of drug serum on expression of $\alpha$-SM-actin of TGF $\beta 1$-induced fibroblasts: Light density values (OD) of normal controls, TGF- $\beta 1$, Captopril and Xuefuzhuyu group were $0.852 \pm 0.0223, \quad 1.071 \pm 0.0486, \quad 0.889 \pm 0.0310$ and $0.858 \pm 0.0204$ respectively, and values of captopril and Xuefuzhuyu group were significantly reduced compared with TGF- $\beta 1$ group $(p<0.01) .3$. Effect of drug serum on mobility of TGF $\beta 1$-induced fibroblasts: Cell migration rates of captopril and Xuefuzhuyu group lowered significantly than that of TGF- $\beta 1$ group $(0.140 \pm 0.0687$ and $0.108 \pm 0.0435$ vs $1.000 \pm 0.0000, \mathrm{p}<0.01)$.

Conclusions 1 . Both captopril and Xuefuzhuyu Capsule inhibit vascular remodelling to a certain extent. Animal experiments shows that more benefits can be obtained from the Xuefuzhuyu than Captopril. 2. Drug serums of two drugs can inhibit activation and migration of adventitial fibriblasts, which may be one of mechanisms of inhibiting vascular remodelling.

\section{e0546 EFFICACY AND SAFETY OF CT-GUIDED PERCUTANEUS CORONARY INTERVENTION OF LEFT MAIN AND OR TRIPLE VESSEL CORONARY ARTERY DISEASE}

doi:10.1136/hrt.2010.208967.546

M Lim, L Devaraja, C de Larrazabal. Singapore Medical Specialists Centre, Paragon Singapore

Introduction Unlike invasive coronary angiography (ICA) which allows visualisation of the coronary vessel lumen, coronary CT angiogram (CCTA) allows both visualisation of the vessel lumen and the vessel wall. The information obtained may potentially allow better pre-PCI planning and safe outcomes.

Aim The aim of this prospective study was to assess the efficacy and safety of CT guided PCI in patients with left main and or triple vessel coronary disease.

Methods Patients, who were listed for PCI based on the results of CCTA, were randomly selected in the study over a 5 year period. Coronary CT angiographic inclusion criteria were, $50 \%-100 \% \mathrm{dm}$ occlusion in a major coronary artery with a target vessel diameter $\geq 2.25 \mathrm{~mm}$, involving all 3 of the main coronary arteries and or left main coronary artery. They underwent CCTA using 64 MSCT and 128 MSCT scans. Coronary stenoses on the ICA were determined independently in the catheterisation laboratory using online quantification software. Coronary angiographic inclusion criteria were $50 \%-100 \%$ diameter occlusion, and vessel diameter $\geq 2.25 \mathrm{~mm}$.

Results A total of 34 consecutive patients who met the inclusion criteria were enrolled during the study period. The mean age was $57.2 \pm 8.2$ years while the male to female ratio was 15:2. There were 544 coronary segments in the study population. There were $177(32.5 \%)$ coronary segments with significant $(\geq 50 \%)$ stenosis on CCTA. In comparison, there were 180 coronary segments with significant stenosis on ICA. The difference is accounted for by 6 false negative and 3 false positive results on CCTA. All patients who were scheduled for PCI on the basis of the CCTA results proceeded to PCI. A total of 116 stents were implanted, representing an average of $3.4 \pm 1.6$ stents per patient (range, $1-12$ stents). Technical success was achieved in all the 34 patients who proceeded to PCI. Significant triple vessel disease was present in $88.2 \%(30 / 34)$ of patients and left main disease was present in $11.8 \%$ (4/34). During PCI and stenting, there were no patients who had a major adverse cardiac event (MACE), developed a stroke, underwent emergency bypass graft surgery or died. During follow-up (mean of 24 months) only 1 patient had another PCI done due to development of significant lesion in the non-stented segment after approximately 38 months from the previous CT scan and PCI. The 5-year event-free survival was $100 \%$.

Conclusion All eligible patients who underwent pre-PCI CT planning were associated with uncomplicated and successful procedures. The long-term outcome was excellent as there were no repeat procedures due to restenosis and no emergency CABG was needed. 